\title{
Modification of the SCA Technique
}

\author{
Nadi Keskin* \\ Dumlupinar University Medical Faculty, Evliya Celebi Training and Research Hospital, Department of Obstetrics and Gynecology, Kutahya, \\ Turkey
}

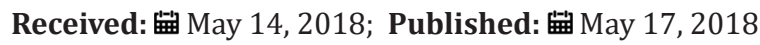

*Corresponding author: Nadi Keskin, Dumlupinar University Medical Faculty, Evliya Celebi Training and Research Hospital, Department of Obstetrics and Gynecology, Okmeydani Street Evliya Celebi Avenue 43000, Kutahya, Turkey, Email: nadikeskin@gmail.com

\section{Editorial}

As you would be appreciate, the rate of cesarean sections increased from $4.5 \%$ to $31.8 \%$ between the years of 1970 and 2007. Although the maternal morbidity and mortality have seriously declined within years due to the implementation of cesarean section, reduction of the complication rates has still considerableimportance, and the revision of the technique and modifications will make contributions to this issue, which involves numerous factors. Therefore, I wanted to describe the lower segment transverse cesarean incision technique, which I have been using with quite a low complication rate for more than ten years. Some of the key points in the currently known and implemented transverse cesarean incision technique are as follows: After entering the abdomen through the suprapubic transverse incision, some surgeons put gauzes into the abdominal cavity for absorption of the blood and the amniotic fluid. Then, the loose peritoneal fold on the upper border of the bladder and the lower anterior uterine segment- bladder flap-is grasped by a haemostatic forceps in the midline. It is cut transversally either with a scalpel or scissors. The scissors are placed between the vesicouterine peritoneum and the myometrium of the lower uterine segment. The scissors are advanced from the midline to the lateral sides. Then, while leaving a space between the scissor tips, the scissors are partially withdrawn. Then, this serosal strip, $2-\mathrm{cm}$ in length, is cut and separated. When the lateral sides are approached, the scissor tips are directed towards the fetal head. The lower flap of the peritoneum is elevated and gently dissected from the underlying myometrium by using blunt and sharp dissection. The depth should not exceed $5 \mathrm{~cm}$, in general. There is a probability of entering the vagina at this point, which is deepened in pregnant women who have suffered from labor pain. The uterus is opened throughout the lower uterine segment at approximately $1 \mathrm{~cm}$ below the upper border of the peritoneal fold. In pregnant women with advanced or complete cervical opening, making the uterine incision from a relatively higher site is important regarding the reduction of the widening probability of the incision laterally towards the uterine arteries together with the unintentional entrance to the vagina. This can be performed by using the guidance of the vesicouterine fold.

For closure of the uterus, the uterine incision is closed by \#0 or \#1 continuous absorbable suture. Most of the surgeons use chromic suture; however, some prefer synthetic absorbable suture. The closure may be single or double-layered; although the conducted studies were not able to show any difference, more studies are needed to be performed on this issue. In Parkland and Alabama Universities, the single-layer closure is preferred. For this purpose, the first suture is placed at the corner, just behind the incision, and then it is continued with locking continuous suture. Each suture should involve the total thickness of the myometrium. The locking continuous suture proceeds to the back of the contra lateral corner. When the lower segment is particularly thin, the edges of the incision can be sufficiently approximated by a single suture layer. The serosal edges have been conventionally approximated by 2-0 chromic catgut suture. Many studies have shown that skipping this step did not lead to any complication. The differences between the cesarean technique using the transverse cesarean incision that "I have been implementing" and the classical method that I have emphasized above are as follows: The abdomen is entered through the suprapubic transverse incision. No gauze is placed into the abdomen. Then, the loose peritoneal fold above the upper border of the bladder and on the lower anterior uterine segmentbladder flap-is grasped by a haemostatic forceps in the midline. It is cut approximately $1 \mathrm{~cm}$ by using scissors, and it is opened bluntly and circumferentially by the fingertips to acquire an edge of approximately $1 \mathrm{~cm}$. The technique is similar thus far; however, from then on, the vesicouterine area is not entered. For this purpose, the peritoneal cut is made from $1-2 \mathrm{~cm}$ above the vesicouterine border. The most important part of the operation is at this point. The most significant difference is the variation that seems to be minor but contributes very significantly to the technique. To be specific, the purpose is to benefit from the flexibility and the strength of the peritoneum while opening the uterus, to prevent the uterine 
incision from extending to the parametrial region and vagina, and to minimize the bladder injuries. Moreover, since the vesicouterine area is not entered and no bladder retractor is used, I have not encountered any bladder injury with this technique which I have been using for more than ten years. I met hematoma formation once or twice. The uterine incision almost never extended to the parametrial region.

The uterine incision starts with a meticulously made shallow incision with a length of $1-2 \mathrm{~cm}$, and by using the blunt scalpel handle, the uterus is quickly entered. Although the most commonly encountered complication is fetal injury while entering the uterus, none occurred in more than 2000 cesarean sections that I have performed using this technique. After entering the uterus, the incision is widened laterally and slightly upwards. Alternatively, since the lower uterine segment is thin, the incision can be enlarged by applying pressure laterally and upwards. The uterine incision should be madeof such a size that the fetal head and body can pass through, without tearing the arteries and veins coursing on the lateral borders of the uterus. Since the visceral peritoneum has not been unnecessarily detached and peeled, it creates a mechanical protective effect and does not allow the incision to extend to the parametrial areas, uterine arteries and veins, at this point. For this reason, I never encountered any hematoma in this region during the cesarean sections that I have been doing for more than ten years. Then, the baby and the placenta are delivered by using the conventional methods; however, at this point, I do not perform the Kristeller maneuver. I only slightly stabilize the fun dally located part of the baby and wait for the baby to come out of the uterus with the help of uterine contractions. Since uncontrolled pressure is not applied to the uterus in this maneuver, it also contributes very significantly to the reduction of uterine ruptures to the parametrial area together with the reduction of hematoma formation. For reduction of the operating time, the corner sutures should be placed with the bottom parts of \#0 Vicryl and should not be cut. Following this suture, the suture put in the other corner is used for the secondlayer closure. Each corner suture is tied to the suture of the contra lateral corner, which was used for closing the layer. This second layer both strengthens the first suture line and reduces the risk of suture dehiscence, providing significant support to hemostasis. The serosal edges have been conventionally approximated by using 2-0 chromic catgut sutures. However, numerous studies have shown that skipping this step did not lead to any complication. In my technique, since the bladder was not previously detached and we are distant from the bladder, minor bleedings from this region can be easily cauterized, and peritoneal sutures are not placed. According to my observation, no significant difference is present between my technique and the conventional technique concerning adhesion formation and pain. However, not using peritoneal sutures shortens the duration of the operation. In my technique, no gauze is placed inside the abdomen before these procedures; I consider that this process increases the rate of intra abdominal adhesions seriously.

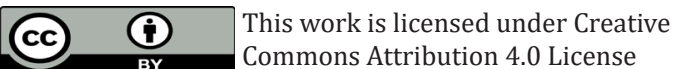

To Submit Your Article Click Here: Submit Article

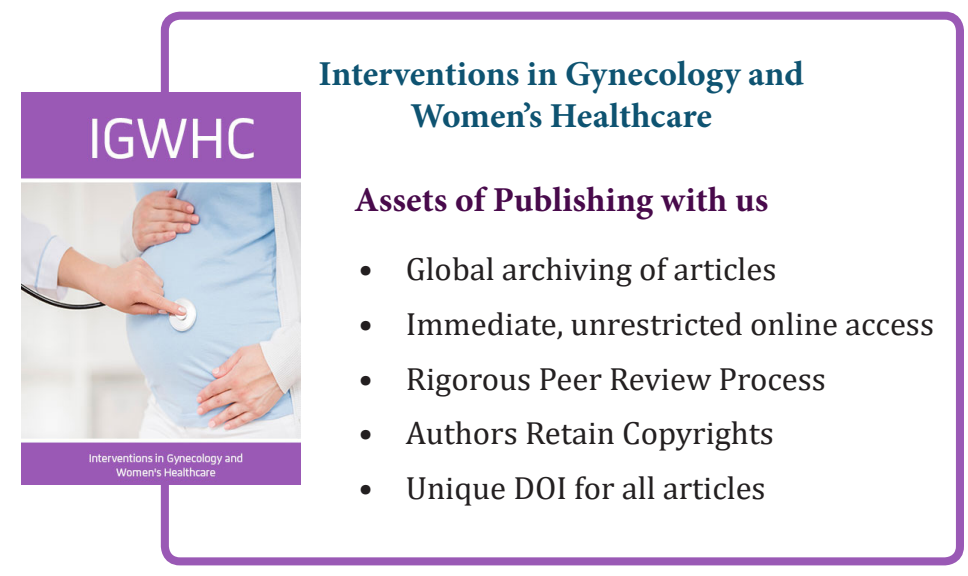

